\title{
Article \\ Impact of Platelet Reactivity in ACS Patients on Clinical Outcomes with Triple Antithrombotic Therapy
}

\author{
Julia Gruttemeier ${ }^{1}$, Yves Cottin ${ }^{1}$, Hermann Yao ${ }^{1}{ }^{\complement}$, Emmanuel De Maistre ${ }^{2}$, Maud Maza ${ }^{1}$, Laurent Bonello ${ }^{3}$, \\ Marc Laine ${ }^{3}$, Noemie Resseguier ${ }^{4}{ }^{-}$, Marianne Zeller ${ }^{5}{ }^{(}$, Laurence Camoin-Jau ${ }^{6}$ and Franck Paganelli ${ }^{3, *}$
}

1 Department of Cardiology, CHU Dijon Bourgogne, 21000 Dijon, France; julia.gruttemeier@sfr.fr (J.G.); yves.cottin@chu-dijon.fr (Y.C.); hermannyao@gmail.com (H.Y.); maud.maza@chu-dijon.fr (M.M.)

2 Department of Biological Haematology, CHU Dijon Bourgogne, 21000 Dijon, France; emmanuel.demaistre@chu-dijon.fr

3 Department of Cardiology, ARCHANTEC, School of Medicine, Aix Marseille University, 13007 Marseille, France; laurent.bonello@ap-hm.fr (L.B.); marc.laine@aphm.fr (M.L.)

4 Public Health, Chronic Diseases and Quality of Life-Research Unit, Aix-Marseille University, 13007 Marseille, France; noemie.RESSEGUIER@univ-amu.fr

5 Team PEC2, EA 7460, Department of Health Sciences, University of Burgundy Franche Comté, 21000 Dijon, France; marianne.zeller@u-bourgogne.fr

6 Department of Biological Haematology, Assistance Publique Hôpitaux de Marseille, Timone Hospital, 13007 Marseille, France; laurence.camoin@ap-hm.fr

* Correspondence: franck.paganelli@ap-hm.fr; Tel.: +33-00-334-9196-8683

check for updates

Citation: Gruttemeier, J.; Cottin, Y.; Yao, H.; De Maistre, E.; Maza, M.; Bonello, L.; Laine, M.; Resseguier, N.; Zeller, M.; Camoin-Jau, L.; et al. Impact of Platelet Reactivity in ACS Patients on Clinical Outcomes with Triple Antithrombotic Therapy. J. Clin Med. 2021, 10, 1565. https://doi.org/ $10.3390 /$ jcm 10081565

Academic Editor: Emmanuel Andrès

Received: 1 February 2021

Accepted: 5 April 2021

Published: 8 April 2021

Publisher's Note: MDPI stays neutral with regard to jurisdictional claims in published maps and institutional affiliations.

Copyright: (c) 2021 by the authors. Licensee MDPI, Basel, Switzerland. This article is an open access article distributed under the terms and conditions of the Creative Commons Attribution (CC BY) license (https:// creativecommons.org/licenses/by/ $4.0 /)$.

\begin{abstract}
Optimal antithrombotic therapy after percutaneous coronary intervention (PCI) in patients on oral anticoagulants (OAC) remains a clinical conundrum. In fact, combining an OAC with dual antiplatelet therapy (triple antithrombotic therapy, TAT) increases the risk of bleeding. Clopidogrel is the only thienopyridine recommended in TAT patients. Whether its response plays a relevant role in this setting remains uncertain. We aimed to evaluate the level of platelet reactivity inhibition (PRI) achieved by oral TAT in Acute Coronary Syndrome (ACS) patients undergoing PCI and its relationship with outcomes. We performed a multicenter prospective observational study and assessed PRI by vasodilator-stimulated phosphoprotein (VASP) index following a loading dose of clopidogrel. The primary endpoint was the incidence of major adverse cerebral or cardiovascular events (MACCE) at six months based on High on Treatment Platelet Reactivity (HTPR, VASP > 50\%). The secondary endpoint was the incidence of bleeding at six months based on Low on Treatment Platelet Reactivity (LTPR, VASP < 16\%). 491 patients were followed up for six months: 7.7\% experienced MACCE and $17.3 \%$ experienced bleeding. There was no significant relationship between HTPR and MACCE, neither between LTPR and bleeding. Vitamin-K antagonist (VKA) treatment was associated with more MACCE and bleeding events, and the majority of events occurred within the first months. VASP index failed to predict outcomes in post-ACS patients with TAT. We confirm that direct acting OAC should be prioritized over VKA in TAT regimen.
\end{abstract}

Keywords: acute coronary syndrome; triple antithrombotic therapy; VASP index; platelet reactivity; clopidogrel

\section{Introduction}

Triple antithrombotic therapy (TAT) (combination of oral anticoagulation, OAC, and dual antiplatelet therapy, DAPT) in the setting of acute coronary syndrome (ACS) is increasingly prescribed because of frequent associated co-morbidities [1]. These patients receive DAPT for the prevention of thrombotic or ischemic events, and oral OAC is recommended in patients with atrial fibrillation $(\mathrm{AF})$, mechanical heart valves and venous thromboembolism [2-5]. Historically, patients who required OAC were systematically excluded from randomized controlled studies (RCT). Data from registries have shown that TAT was effective in reducing ischemic events, but with significantly increased risk of 
bleeding $[3,6,7]$. As the only thienopyridine recommended in recent guidelines for the TAT regimen, clopidogrel has been shown through platelet function assessment test (PFT) to have a high degree of inter-individual variability for platelet reactivity inhibition (PRI) [8] High on-treatment platelet reactivity (HTPR) is associated with ischemic events and low on-treatment platelet reactivity (LTPR) is associated with bleeding $[8,9]$. Thus, the use of clopidogrel and individual variability between individuals (such as obesity, impaired kidney function) may make it difficult to implement TAT [9]. To address this issue, we aimed to investigate the inter-individual variability for PRI in ACS patients undergoing percutaneous coronary intervention (PCI) and receiving TAT regimen in order to identify sub-groups at higher risk populations for bleeding or ischemic events regarding HTPR or LTPR status.

\section{Materials and Methods}

\subsection{Study Design}

We conducted a multicenter observational prospective study from January 2018 to December 2019. We selected patients aged 18-90 years, with preexisting indication for OAC, admitted for ACS and who underwent PCI with stent implantation [3]. Patients receiving TAT regimen including clopidogrel for less than $48 \mathrm{~h}$, patients who have been treated with ticagrelor or prasugrel for at least $24 \mathrm{~h}$ prior to PRI evaluation, and patients unavailable for PRI evaluation (holidays, weekends, etc.) were not considered in our study. Exclusion criteria were: history of intracranial bleeding, cardiogenic shock, contraindication for aspirin or clopidogrel in the previous six months, thrombocytopenia, major bleeding in the past month, pregnancy, concomitant severe illness with expected survival $<1$ month, surgery within one month, liver failure. All patients undergoing PCI gave an informed consent before inclusion in the study. Patients were followed up for six months. The protocol was approved by local ethic committees (CPP "comité de protection des personnes" and ANSM "agence nationale de sécurité du médicament et des produits de santé") and in accordance with the declaration of Helsinki [Prospective Real-world Registry Describing Treatment Regimens (PRAETORIAN) NCT03942913].

\subsection{Periprocedural Antithrombotic Management}

During PCI, additional low-dose of parenteral anticoagulation (bolus of unfractionated heparin $100 \mathrm{IU} / \mathrm{kg} \mathrm{UHF}$ ) was administered in all dabigatran-treated patients [10]. In other cases, $\mathrm{PCI}$ was performed without interruption of OAC and without additional anticoagulation, in accordance with the guidelines [3]. Additional UFH should be administered as per usual practice to support $\mathrm{PCI}$, at standard dose $(70$ to $100 \mathrm{U} / \mathrm{Kg})$ in patients receiving DOAC (direct oral anticoagulant) and reduced dose (30 to $50 \mathrm{U} / \mathrm{Kg}$ ) in case of vitamin-K antagonist (VKA) therapy [3]. All patients received a bolus of $250 \mathrm{mg}$ aspirin i.v. and a loading dose of $600 \mathrm{mg}$ clopidogrel just before the PCI followed by daily DAPT regimen. In patients already on OAC, continuation of treatment with the same agent after PCI was encouraged, particularly if there was good compliance, good control of international normalized ratio (INR), and in patients free of ischemic and hemorrhagic events.

\subsection{Risk Score, International Normalized Ratio, Dosing Regimen of a DOAC for Combination Therapy in TAT}

CHA2DS2VASc scores reflect the risk of stroke among patients with AF who are not receiving anticoagulant therapy. HAS-BLED scores reflect the risk of bleeding among patients with AF who are receiving anticoagulant therapy [3]. For all patients who received VKA, the predicted INR was set between 2 and 2.5 [3]. Consistent with a recent review [3], DOAC was prescribed at full stroke prevention doses guidelines for AF (150 mg bid for Dabigatran, $5 \mathrm{mg}$ bid for Apixaban). If rivaroxaban was used, the $15 \mathrm{mg}$ once-daily dose (e.g., rather than the $20 \mathrm{mg}$ dose tested in trials) was considered a reasonable alternative [3]. Because of DOAC short half-life and limited drug-drug interactions, blood level tests were not needed. In some situations, the issue of blood testing for DOAC levels arises but there 
is a consensus suggesting that it would not be an appropriate tool for the management of TAT patients [3].

\subsection{Platelet Reactivity Measurements}

PRI Measurements has been previously described. Blood samples were drawn at least $6 \mathrm{~h}$ and within $24 \mathrm{~h}$ after clopidogrel loading dose [11]. Briefly, a citrated blood sample was incubated with prostaglandin E1 (PGE1) or with PGE1 and ADP $10 \mu \mathrm{mol} / \mathrm{L}$ for $10 \mathrm{~min}$ and fixed with paraformaldehyde, after which the platelets were permeabilized with nonionic detergent. Analyses were performed on an EPICS XL-MCL flow cytometer (Beckman Coultronics, Margency, France). HTPR was defined as a VASP index $\geq 50 \%$ and LTPR as a VASP index $<16 \%[12,13]$.

\subsection{Outcomes}

The primary efficacy endpoint was major adverse cardiac or cerebrovascular events (MACCE) at six months (composite of all-cause mortality, non-procedural myocardial infarction, any urgent coronary revascularization, and ischemic stroke and/or extracranial thromboembolism). Deaths from vascular disease were defined as cardiovascular or cerebrovascular death and any death with unknown cause. New myocardial infarction and stroke have been previously defined and described [14,15]. Systemic embolism was diagnosed as acute arterial obstruction of the limbs or any organ and confirmed by angiography.

The secondary safety end point at six months was the occurrence of clinically significant bleeding (major bleeding, mild or moderate bleeding) according to Thrombolysis in Myocardial Infarction (TIMI) criteria or bleeding requiring medical attention [16].

\subsection{Data Collection}

Follow-up data after discharge was obtained during routine scheduled outpatient visits at one month and during telephone calls to patients every three months according to the clinical evolution. Standardized questions were used to assess bleeding episodes, thrombotic events and use of medications.

\subsection{Patient and Public Involvement}

For each patient, the investigator presented the advantages and disadvantages of the medical therapy and discussed the benefits of his participation to this study. Then, a consent form was signed in the presence of the investigator.

\subsection{Statistical Analyses}

Continuous data are expressed as mean (standard deviation) or median (interquartile range), and categorical data as numbers and percentages. Characteristics of the patients were described and compared according to the VASP index (cut-off: $50 \%$ or $16 \%$ ). Statistical comparisons between groups used Student's $t$-tests or Mann-Whitney tests for continuous variables, and chi-square test or Fisher exact test for categorical variables. The Cox regression model was used to estimate hazard ratios and their $95 \%$ confidence intervals. We used RStudio software version 1.2.5019 (2009-2019 RStudio, Inc., Boston, MA, USA). $p$ values $<0.05$ were considered significant.

\section{Results}

\subsection{Population}

From PRAETORIAN registry, 765 consecutive patients receiving long-term OAC and PCI were assessed for eligibility. Withdrawn informed consent was found in 25 patients, 67 patients were lost to follow-up, PRI was not performed in 182 patients and these were excluded from the statistical analyses. A total of 491 patients were studied with a follow-up of six months (Figure 1). Tables 1 and 2 summarizes the characteristics of the population. Of the 491 patients included, $72 \%$ were male and the median age was 78 years. Two 
hundred and seven patients (42.2\%) had a history of CAD, and 99 had a history of bleeding $(20 \%)$ including cerebral $(0.8 \%)$ and gastrointestinal $(7.5 \%)$ hemorrhages. The median $\mathrm{CHA}_{2} \mathrm{DS}_{2}$ VASc score was $4.72(4-6)$ and the median HAS-BLED score was $2.52(2,3)$. All patients were hospitalized for ACS, in most cases of NSTEMI; $99 \%$ of patients underwent PCI with stent implantation. Indication for OAC was mainly AF (75.7\%), including $84.3 \%$ with prior history of AF and $15.7 \%$ with new-onset AF. Oral anticoagulant was VKA in 201 patients $(41 \%)$, and DOAC in 290 patients $(22.8 \%$ rivaroxaban, $22 \%$ apixaban and $14.2 \%$, dabigatran). The median PRI score was 49\% (25-69), but 250 patients (50.9\%) had a score $>50 \%$, reflecting poor response to clopidogrel therapy. LTPR was found in 39 patients $(7.9 \%)$. In addition, the mean length of triple therapy was 3.51 months, which is in accordance with the recommendations at the time of the start of the study.

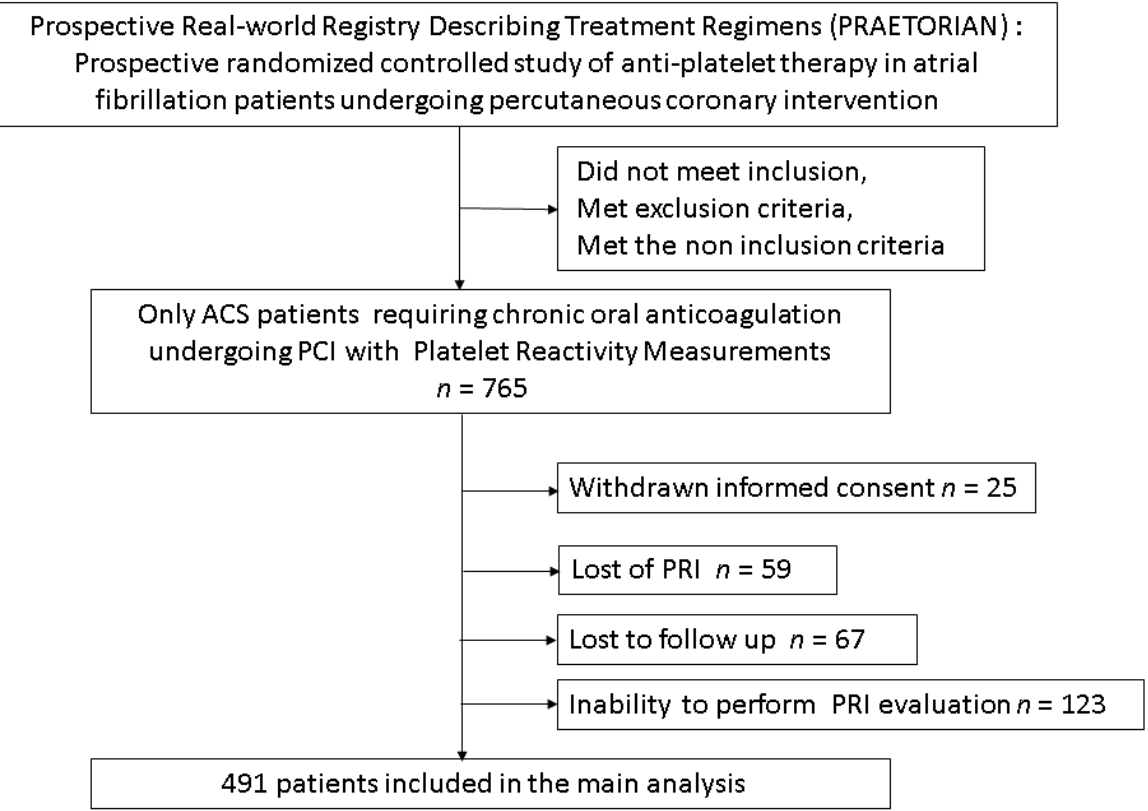

Figure 1. Flow-chart. ACS: acute coronary syndrome; PCI: percutaneous coronary intervention; PRI: platelet reactivity inhibition.

Table 1. Baseline characteristics for patients with six months follow-up $(N=491)$.

\begin{tabular}{lc}
\hline Variable & $N=\mathbf{4 9 1}(\mathbf{\%})$ \\
\hline Female & $137(28)$ \\
Age, year & 78.4 \\
Hypertension & $353(72)$ \\
Dyslipidemia & $391(79)$ \\
Smoker & $147(30)$ \\
Diabetes & \\
None & $300(60.8)$ \\
T1DM & $24(4.8)$ \\
T2DM & $167(34)$ \\
History of vascular disease & $436(89)$ \\
History of heart failure & $197(40.1)$ \\
History of hemorrhage & $99(20.1)$ \\
$\quad$ None & $392(80)$ \\
ENT & $17(3.4)$ \\
Cerebral & $4(0.8)$ \\
Digestive & $37(7.5)$ \\
Urinary & $11(2.2)$ \\
Hematoma & $19(3.8)$ \\
Vascular & $11(2.2)$ \\
\hline
\end{tabular}


Table 1. Cont.

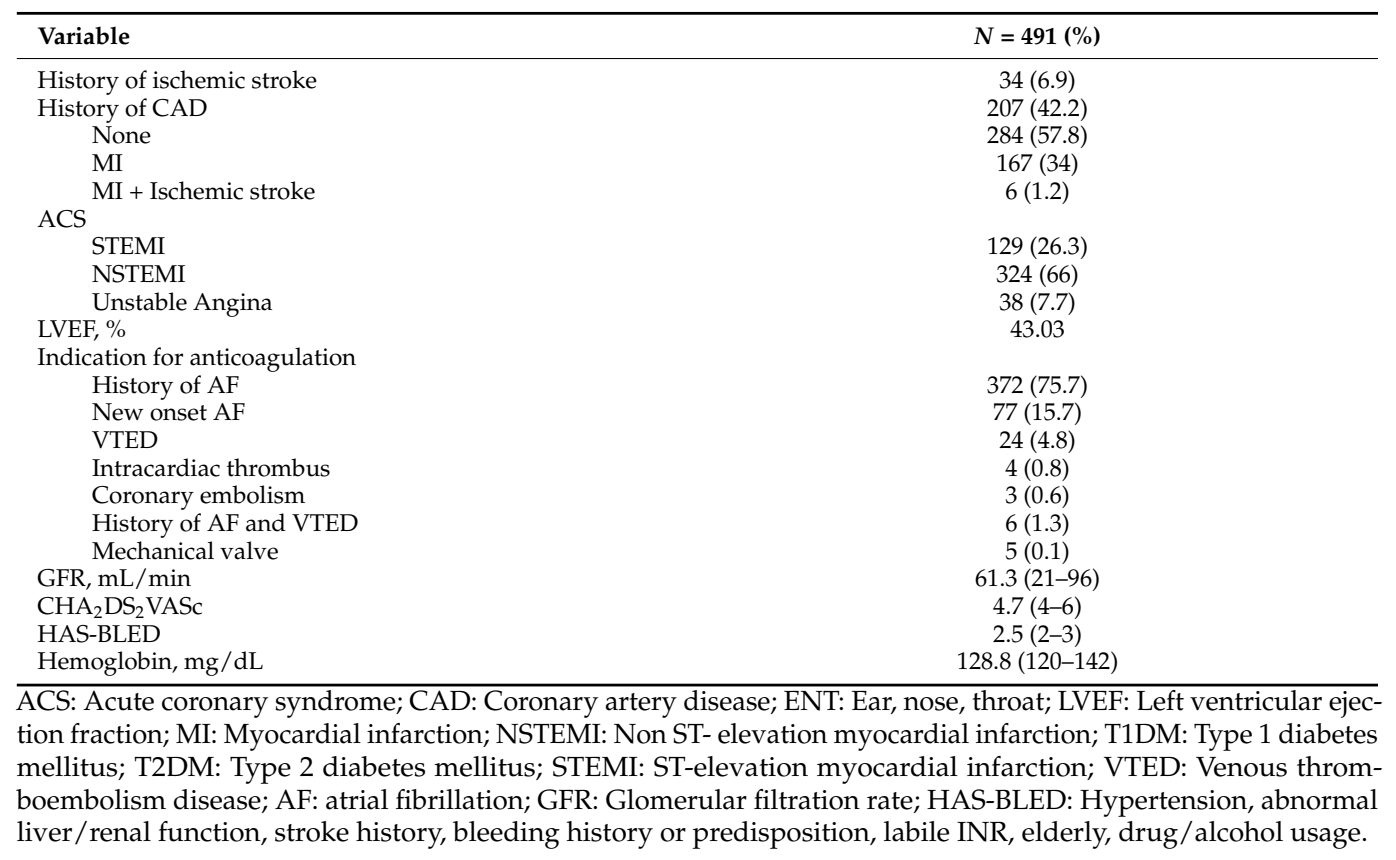

Table 2. Indication for anticoagulation, major adverse cardiac or cerebrovascular events and bleedings for patients with six months follow-up $(N=491)$.

\begin{tabular}{|c|c|}
\hline Variable & $N=491(\%)$ \\
\hline \multicolumn{2}{|l|}{ Indication for anticoagulation } \\
\hline History of AF & $372(75.7)$ \\
\hline New onset AF & $77(15.7)$ \\
\hline VTED & $24(4.8)$ \\
\hline Intracardiac thrombus & $4(0.8)$ \\
\hline Coronary embolism & $3(0.6)$ \\
\hline History of AF and VTED & $6(1.3)$ \\
\hline Mechanical valve & $5(0.1)$ \\
\hline \multicolumn{2}{|c|}{ Triple therapy: aspirin+ clopidogrel associated with } \\
\hline VKA & $201(41)$ \\
\hline Rivaroxaban & $112(22.8)$ \\
\hline Apixaban & $108(22)$ \\
\hline Dabigatran & $70(14.2)$ \\
\hline VASP, $\%$ & $49(25-69)$ \\
\hline INR dosage & $\mathrm{N}$ \\
\hline Under dosing INR & $111(55)$ \\
\hline Targeted INR & $80(40)$ \\
\hline Over-dosing INR & $10(5)$ \\
\hline Length of triple therapy in months & $3.51(1-6)$ \\
\hline PPI during triple therapy & $455(92.6)$ \\
\hline \multicolumn{2}{|l|}{ Revascularization } \\
\hline No PCI & $2(0.4)$ \\
\hline PCI + stent & $487(99.2)$ \\
\hline PCI without stent & $2(0.4)$ \\
\hline Follow-up, days & 190 \\
\hline MACCE with triple therapy & $38(7.7)$ \\
\hline None & $453(92.2)$ \\
\hline Non-fatal MI & $16(3.2)$ \\
\hline Ischemic stroke & $2(0.5)$ \\
\hline Death & $18(3.6)$ \\
\hline
\end{tabular}


Table 2. Cont.

\begin{tabular}{|c|c|}
\hline Variable & $N=491(\%)$ \\
\hline Urgent revascularization & $2(0.5)$ \\
\hline MACCE on TAT regimen & $38(7.7)$ \\
\hline VKA & $18(3.6)$ \\
\hline Rivaroxaban & $14(2.8)$ \\
\hline Apixaban & $3(0.6)$ \\
\hline Dabigatran & $3(0.6)$ \\
\hline MACCE on rivaroxaban & $14(2.6)$ \\
\hline Non-fatal MI & $8(1.6)$ \\
\hline Death & $4(0.8)$ \\
\hline Ischemic stroke & $0(0)$ \\
\hline Urgent revascularization & $2(0.4)$ \\
\hline MACCE on apixaban & $3(0.6)$ \\
\hline Non-fatal MI & $3(0.6)$ \\
\hline Death & $0(0)$ \\
\hline Ischemic stroke & $0(0)$ \\
\hline Urgent revascularization & $0(0)$ \\
\hline MACCE on dabigatran & $3(0.6)$ \\
\hline Non-fatal MI & $3(0.6)$ \\
\hline Death & $0(0)$ \\
\hline Ischemic stroke & $0(0)$ \\
\hline Urgent revascularization & $0(0)$ \\
\hline MACCE on VKA & $18(3.7)$ \\
\hline Non-fatal MI & $2(0.4)$ \\
\hline Death & $14(2.8)$ \\
\hline Ischemic stroke & $2(0.4)$ \\
\hline Urgent revascularization & $0(0)$ \\
\hline Bleeding with triple therapy & $85(17.3)$ \\
\hline None & $406(82.2)$ \\
\hline Cerebral & $4(0.8)$ \\
\hline VKA & $2(0.4)$ \\
\hline Rivaroxaban & $1(0.2)$ \\
\hline Dabigatran & $1(0.2)$ \\
\hline Apixaban & $0(0)$ \\
\hline Digestive & $38(7.8)$ \\
\hline VKA & $14(2.8)$ \\
\hline Rivaroxaban & $18(3.6)$ \\
\hline Apixaban & $4(0.8)$ \\
\hline Dabigatran & $2(0.4)$ \\
\hline Hemoptysis & $7(1.5)$ \\
\hline VKA & $3(0.6)$ \\
\hline Rivaroxaban & $1(0.2)$ \\
\hline Apixaban & $1(0.2)$ \\
\hline Dabigatran & $2(0.2)$ \\
\hline Urinary & $11(2.3)$ \\
\hline VKA & $9(1.8)$ \\
\hline Rivaroxaban & $0(0)$ \\
\hline Apixaban & $1(0.2)$ \\
\hline Dabigatran & $1(0.2)$ \\
\hline Hematoma & $13(2.6)$ \\
\hline VKA & $10(2)$ \\
\hline Rivaroxaban & $1(0.2)$ \\
\hline Apixaban & $1(0.2)$ \\
\hline Dabigatran & $1(0.2)$ \\
\hline Vascular & $10(2)$ \\
\hline VKA & $4(0.8)$ \\
\hline Rivaroxaban & $1(0.2)$ \\
\hline
\end{tabular}


Table 2. Cont.

\begin{tabular}{cc}
\hline Variable & $N=4 \mathbf{9 1}$ (\%) \\
\hline Apixaban & $0(0)$ \\
Dabigatran & $5(1.0)$ \\
ENT & $2(0.4)$ \\
VKA & $0(0)$ \\
Rivaroxaban & $0(0)$ \\
Apixaban & $0(0)$ \\
Dabigatran & $2(0.4)$ \\
TIMI Class & $85(17.3)$ \\
Major & $6(1.2)$ \\
Minor & $40(8.1)$ \\
Minimal & $39(7.9)$ \\
Bleeding on TT regimen & $85(17.3)$ \\
VKA & $42(8.5)$ \\
Major & $4(0.8)$ \\
Minor & $20(4)$ \\
Minimal & $18(3.7)$ \\
Rivaroxaban & $22(4.5)$ \\
Major & $2(0.4)$ \\
Minor & $15(3.1)$ \\
Minimal & $5(1)$ \\
Apixaban & $7(1.8)$ \\
Major & $0(0)$ \\
Minor & $3(0.6)$ \\
Minimal & $4(1.2)$ \\
Dabigatran & $12(2.4)$ \\
Major & $0(0)$ \\
Minor & $2(0.4)$ \\
Minimal & $12(2)$ \\
\hline AF: Atriabilat \\
Ming
\end{tabular}

AF: Atrial fibrillation; INR: International normalized ratio; MACCE: Major adverse cardiac and cerebrovascular events; MI: Myocardial infarction; PCI: Percutaneous coronary intervention; PPI: proton pump inhibitor; VASP: Vasodilator-stimulated phosphoprotein; TIMI: Thrombolysis in myocardial infarction; VKA: Vitamin K antagonist; VTED: Venous thromboembolism disease; TT: Triple therapy.

\subsection{Major Adverse Cerebral and Cardiovascular Events at 6 Months}

At six months follow-up, 38 patients experienced MACCE (7.7\%). Sixteen had a recurrence of ACS (3.6\%) and 18 died (3.7\%). Two patients had an ischemic stroke (0.5\%). Ischemic events were more frequent in the VKA group (Table 2).

\subsection{Bleedings Events at Six Months}

At six months, a TIMI bleeding event occurred in 85 patients (17.3\%) receiving TAT regimen. Main bleedings were gastro-intestinal (44.7\%) and $49 \%$ occurred in the VKA group. There were six major bleeds, 40 moderate, and 39 mild (Table 2). The effects of the different DOAC on ischemic and bleeding events are detailed in Table 2. No medical history or cardiovascular risk could be linked to the onset of bleeding or MACCE. The cumulative incidence of MACCE and bleeding at six months was highest among patients who had been assigned to receive VKA and lowest among those assigned to receive apixaban.

Based on the increasing evidence suggesting an association between HTPR and ischemic events, and between LTPR and bleeding events, we performed a further analysis on the occurrence of MACCE and bleedings regarding HTPR or LTPR status [11].

\subsection{Bleeding and MACCE on HTPR or LTPR Group}

Of all patients, $250(51 \%)$ had a HTPR (VASP > 50\%) with a high proportion of diabetics, and a higher prescription rate of DOAC (Table 3). 
Table 3. Baseline characteristics for patients with 6 months follow-up, VASP cut-off 50\% ( $\mathrm{N}=491)$.

\begin{tabular}{|c|c|c|c|}
\hline Variable & $\begin{array}{c}\text { VASP } \leq 50 \% \\
N=241\end{array}$ & $\begin{array}{c}\text { VASP > 50\% } \\
N=250\end{array}$ & $p$ \\
\hline Female & $76(32)$ & $61(24)$ & NS \\
\hline Age, year & 79 & 78 & NS \\
\hline Hypertension & $170(70.5)$ & $183(73)$ & NS \\
\hline Dyslipidemia & $185(76)$ & $206(82)$ & NS \\
\hline Smoker & $69(28)$ & $78(32)$ & NS \\
\hline \multicolumn{4}{|l|}{ Diabetes } \\
\hline None & $137(56)$ & $163(65)$ & 0.037 \\
\hline T1DM & $17(7)$ & $7(3)$ & \\
\hline $\mathrm{T} 2 \mathrm{DM}$ & $87(36)$ & $80(31)$ & \\
\hline History of vascular disease & $210(87)$ & $226(90)$ & NS \\
\hline History of heart failure & $85(35)$ & $112(44)$ & NS \\
\hline History of hemorrhage & $44(18.2)$ & $55(22)$ & NS \\
\hline ENT & $6(2.4)$ & $11(4.4)$ & \\
\hline Cerebral & $2(0.8)$ & $2(0.8)$ & \\
\hline Digestive & $20(8.2)$ & $17(6.8)$ & \\
\hline Urinary & $4(1.7)$ & $7(2.8)$ & \\
\hline Hematoma & $8(3.3)$ & $11(4.4)$ & \\
\hline Vascular & $4(1.6)$ & $7(2.8)$ & \\
\hline History of ischemic stroke & $11(44)$ & $23(9.2)$ & 0.043 \\
\hline History of CAD & $104(43)$ & $103(42)$ & NS \\
\hline None & $137(57)$ & $147(58)$ & \\
\hline MI & $90(37)$ & $77(30.8)$ & \\
\hline $\mathrm{MI}+$ Ischemic stroke & $3(1.2)$ & $3(1.2)$ & \\
\hline ACS & & & NS \\
\hline STEMI & $79(32.7)$ & $50(20)$ & \\
\hline NSTEMI & $152(63)$ & $172(68.2)$ & \\
\hline Unstable Angina & $10(4.1)$ & $28(11.2)$ & \\
\hline LVEF, \% & $41(35-59)$ & $45(35-60)$ & NS \\
\hline Indication for anticoagulation & & & NS \\
\hline History of AF & $185(76)$ & $187(75)$ & \\
\hline Newonset AF & $45(18.6)$ & $32(12.8)$ & \\
\hline VTED & $5(2)$ & $19(7.6)$ & \\
\hline Intracardiac thrombus & $1(0.4)$ & $3(1.2)$ & \\
\hline Coronary embolism & $1(0.4)$ & $2(1)$ & \\
\hline History of AF and VTED & $1(0.4)$ & $3(2)$ & \\
\hline Mechanical valve & $3(1.2)$ & $2(1)$ & \\
\hline $\mathrm{GFR}, \mathrm{mL} / \mathrm{min}$ & 56.88 & 62.09 & 0.016 \\
\hline $\mathrm{CHA}_{2} \mathrm{DS}_{2} \mathrm{VASc}$ & $5.3(4-6)$ & $4.4(4-5)$ & NS \\
\hline HAS-BLED & $2.6(2-3)$ & $2.45(2-3)$ & NS \\
\hline Hemoglobin, mg/dL & $123(121-140)$ & $135(120-143)$ & NS \\
\hline
\end{tabular}

VASP: Vasodilator-stimulated phosphoprotein; ACS: Acute coronary syndrome; CAD: Coronary artery disease ENT: Ear, nose, throat; LVEF: Left ventricular ejection fraction; MI: Myocardial infarction; NSTEMI: Non STelevation myocardial infarction; T1DM: Type 1 diabetes mellitus; T2DM: Type 2 diabetes mellitus; STEMI: ST-elevation myocardial infarction; VTED: Venous thromboembolism disease; GFR: Glomerular filtration rate; HAS-BLED: Hypertension, abnormal liver/renal function, stroke history, bleeding history or predisposition, labile INR, elderly, drug/alcohol usage.

No difference was found in the HTPR group regarding MACCE, but conversely with a higher number of bleeding events (Table 4). 
Table 4. Indication for anticoagulation, major adverse cardiac or cerebrovascular events and bleeding for patients with six months follow-up, VASP cut-off $50 \%(N=491)$.

\begin{tabular}{|c|c|c|c|}
\hline Variable & $\begin{array}{c}\text { VASP } \leq 50 \% \\
N=241\end{array}$ & $\begin{array}{c}\text { VASP }>50 \% \\
N=250\end{array}$ & $p$ \\
\hline \multicolumn{4}{|l|}{$\begin{array}{l}\text { Triple therapy: aspirin+ } \\
\text { clopidogrel associated with }\end{array}$} \\
\hline VKA & $110(45.6)$ & $91(36.4)$ & \\
\hline Rivaroxaban & $52(21.6)$ & $60(24)$ & \\
\hline Apixaban & $48(19.9)$ & $60(24)$ & \\
\hline Dabigatran & $31(13.3)$ & $39(15.2)$ & \\
\hline VASP, $\%$ & $27.5(22-37)$ & $75(64-76)$ & $<0.001$ \\
\hline INR dosage & & & NS \\
\hline Under dosing INR & $51(50)$ & $60(59)$ & \\
\hline Targeted INR & $51(44)$ & $39(35)$ & \\
\hline Over-dosing INR & $9(8)$ & $1(1)$ & \\
\hline $\begin{array}{l}\text { Length of triple therapy in } \\
\text { months }\end{array}$ & $3.5(1-6)$ & $3.6(1-6)$ & NS \\
\hline PPI during triple therapy & $230(95)$ & $225(90)$ & NS \\
\hline Revascularization & & & NS \\
\hline No PCI & $0(0)$ & $2(0.4)$ & \\
\hline $\mathrm{PCI}+$ stent & $241(100)$ & $246(98.4)$ & \\
\hline PCI without stent & $0(0)$ & $2(0.8)$ & \\
\hline Follow-up, days & $190(190-190)$ & $190(190-190)$ & NS \\
\hline MACCE with triple therapy & $23(9.5)$ & $15(6)$ & NS \\
\hline Non-fatal MI & $9(3.7)$ & $7(2.8)$ & \\
\hline Ischemic stroke & $1(0.4)$ & $1(0.4)$ & \\
\hline Death & $11(4.6)$ & $7(2.8)$ & \\
\hline Urgent revascularization & $2(0.8)$ & $0(0)$ & \\
\hline MACCE on TAT regimen & $23(9.5)$ & $15(6)$ & NS \\
\hline VKA & $10(4.1)$ & $8(3.2)$ & \\
\hline Rivaroxaban & $10(4.1)$ & $4(1.6)$ & \\
\hline Apixaban & $1(0.4)$ & $2(0.8)$ & \\
\hline Dabigatran & $2(0.8)$ & $1(0.4)$ & \\
\hline Bleeding with triple therapy & $33(13.6)$ & $52(20.8)$ & 0,04 \\
\hline Cerebral & $2(0.8)$ & $2(0.8)$ & \\
\hline Digestive & $12(5)$ & $26(10.4)$ & \\
\hline Hemoptysis & $3(1.2)$ & $4(1.6)$ & \\
\hline Urinary & $2(0.8)$ & $9(3.6)$ & \\
\hline Hematoma & $11(4.6)$ & $2(0.8)$ & \\
\hline Vascular & $1(0.4)$ & $9(3.6)$ & \\
\hline ENT & $2(0.8)$ & $0(0)$ & \\
\hline TIMI class & $33(13.6)$ & $52(20.8)$ & 0.04 \\
\hline Major & $3(1.2)$ & $3(1.2)$ & \\
\hline Minor & $15(6.2)$ & $25(10)$ & \\
\hline Minimal & $15(6.2)$ & $24(10)$ & \\
\hline Bleeding on TAT regimen & $33(13.6)$ & $52(20.8)$ & 0.04 \\
\hline VKA & $19(7.8)$ & $23(9.2)$ & \\
\hline Rivaroxaban & $11(4.5)$ & $11(4.8)$ & \\
\hline Apixaban & $0(1)$ & $7(2.4)$ & \\
\hline Dabigatran & $3(0.5)$ & $11(4.4)$ & \\
\hline
\end{tabular}

AF: Atrial fibrillation; INR: International normalized ratio; MACCE: Major adverse cardiac and cerebrovascular events; MI: Myocardial infarction; PCI: Percutaneous coronary intervention; PPI: proton pump inhibitor; VASP: Vasodilator-stimulated phosphoprotein; TIMI: Thrombolysis in myocardial infarction; VKA: Vitamin K antagonist; VTED: Venous thromboembolism disease; TAT; triple antithrombotic therapy; ENT: Ear, nose, throat.

Only $8 \%(N=39)$ had a LTPR with no increase in bleeding events. The characteristics of the patients at baseline were well balanced among the two groups except for more prescription of VKA agent in VASP $>16 \%$ group (Tables 5 and 6 ). 
Table 5. Baseline characteristics for patients with six months follow-up, VASP cut-off $16 \%(N=491)$.

\begin{tabular}{|c|c|c|c|}
\hline Variable & $\begin{array}{c}\text { VASP } \leq 16 \% \\
N=39\end{array}$ & $\begin{array}{c}\text { VASP }>16 \% \\
\quad N=452\end{array}$ & $p$ \\
\hline Female & $12(30.7)$ & $125(27.7)$ & NS \\
\hline Age, year & 79.1 & 78.3 & NS \\
\hline Hypertension & $27(69.2)$ & $326(72.1)$ & NS \\
\hline Dyslipidemia & $28(71.7)$ & $363(80.3)$ & NS \\
\hline Smoker & $11(28.2)$ & $136(30)$ & NS \\
\hline Diabetes & & & NS \\
\hline None & $20(51.2)$ & $280(61.9)$ & NS \\
\hline T1DM & $3(7.7)$ & $21(4.6)$ & \\
\hline T2DM & $16(41)$ & $151(34.7)$ & \\
\hline History of vascular disease & $31(79)$ & $405(89.6)$ & NS \\
\hline History of heart failure & $12(30.7)$ & $185(40.9)$ & NS \\
\hline History of hemorrhage & $10(25)$ & 89 (19) & NS \\
\hline ENT & $1(2.5)$ & $16(3.5)$ & \\
\hline Cerebral & $2(5)$ & $2(0.4)$ & \\
\hline Digestive & $2(5)$ & $35(7.7)$ & \\
\hline Urinary & $2(5)$ & 9 (é) & \\
\hline Hematoma & $2(5)$ & 17 (3.è) & \\
\hline Vascular & $1(2.5)$ & $10(2.2)$ & \\
\hline History of ischemic stroke & $5(12)$ & $29(6.4)$ & NS \\
\hline History of CAD & $13(33)$ & $194(43)$ & NS \\
\hline None & 26 & 258 & \\
\hline MI & $07(18)$ & $160(36)$ & \\
\hline $\mathrm{MI}+$ Ischemic stroke & $1(3)$ & $5(1.1)$ & \\
\hline ACS & 39 & 452 & NS \\
\hline STEMI & $15(38.5)$ & $114(25.2)$ & \\
\hline NSTEMI & $20(51.2)$ & $304(67.2)$ & \\
\hline Unstable Angina & $4(10.2)$ & $34(7.5)$ & \\
\hline LVEF, \% & $43.92(35-59)$ & $41.3(35-60)$ & NS \\
\hline $\mathrm{GFR}, \mathrm{mL} / \mathrm{min}$ & 57.79 & 59.09 & NS \\
\hline $\mathrm{CHA}_{2} \mathrm{DS}_{2} \mathrm{VASc}$ & $4.67(4-5)$ & $5.23(4-6)$ & 0.02 \\
\hline HAS-BLED & $2.5(2-3)$ & $2.72(2-3)$ & NS \\
\hline Hemoglobin, mg/dL & $127(121-140)$ & $131(120-143)$ & NS \\
\hline
\end{tabular}

VASP: Vasodilator-stimulated phosphoprotein; ACS: Acute coronary syndrome; CAD: Coronary artery disease ENT: Ear, nose, throat; LVE: Left ventricular ejection fraction; MI: Myocardial infarction; NSTEMI: Non STelevation myocardial infarction; T1DM: Type 1 diabetes mellitus; T2DM: Type 2 diabetes mellitus; STEMI: STelevation myocardial infarction; GFR: Glomerular filtration rate; HAS-BLED: Hypertension, abnormal liver/renal function, stroke history, bleeding history or predisposition, labile INR, elderly, drug/alcohol usage.

Table 6. Indication for anticoagulation, major adverse cardiac or cerebrovascular events and bleedings for patients with six months follow-up, VASP cut-off $16 \%(N=491)$.

\begin{tabular}{lccc}
\hline Variable & $\begin{array}{c}\text { VASP } \leq \mathbf{1 6} \% \\
\mathbf{N}=\mathbf{3 9}\end{array}$ & $\begin{array}{c}\text { VASP }>\mathbf{1 6} \% \\
\mathbf{N}=\mathbf{4 5 2}\end{array}$ & $p$ \\
\hline $\begin{array}{l}\text { Triple therapy: aspirin+ } \\
\text { clopidogrel associated with }\end{array}$ & & & 0.002 \\
VKA & $13(33.3)$ & $188(41.5)$ & \\
Rivaroxaban & $7(18)$ & $105(22.2)$ & \\
Apixaban & $10(25.6)$ & $98(21.7)$ & \\
Dabigatran & $09(23)$ & $61(13.5)$ & $<0.001$ \\
VASP, $\%$ & $9.5(10-16)$ & $53(64-76)$ & NS \\
INR dosage & $5(38.2)$ & $106(56.4)$ & \\
$\quad$ Under dosing INR & $4(30.4)$ & $76(40.4)$ & \\
Targeted INR & $4(30.4)$ & $6(3.1)$ & NS \\
Over-dosing INR & $3.72(1-6)$ & $3.58(1-6)$ & \\
Length of triple therapy in months & &
\end{tabular}


Table 6. Cont.

\begin{tabular}{|c|c|c|c|}
\hline Variable & $\begin{array}{c}\text { VASP } \leq 16 \% \\
N=39\end{array}$ & $\begin{array}{c}\text { VASP }>16 \% \\
N=452\end{array}$ & $p$ \\
\hline PPI during triple therapy & $35(89)$ & $42(93)$ & NS \\
\hline Revascularization & & & NS \\
\hline No PCI & 1 & $1(0.2)$ & \\
\hline PCI + stent & $37(99)$ & $450(99.6)$ & \\
\hline PCI without stent & 1 & $1(0.2)$ & \\
\hline Follow-up, days & $190(190-190)$ & $190(190-190)$ & NS \\
\hline MACCE with triple therapy & $4(10.2)$ & $34(7.6)$ & NS \\
\hline Non-fatal MI & $1(2.5)$ & $15(3.3)$ & \\
\hline Ischemic stroke & $1(2.5)$ & $1(0.30)$ & \\
\hline Death & $1(2.5)$ & $17(3.7)$ & \\
\hline Fatal MI & $1(2.5)$ & $1(0.3)$ & \\
\hline MACCE on TT regimen & $4(10.2)$ & $34(7.6)$ & NS \\
\hline VKA & $2(5.1)$ & $16(3.5)$ & \\
\hline Rivaroxaban & $2(5.1)$ & $12(1.8)$ & \\
\hline Apixaban & $0(0)$ & $3(0.7)$ & \\
\hline Dabigatran & $0(0)$ & $3(1.5)$ & \\
\hline Bleeding with triple therapy & $6(15.4)$ & $79(17.5)$ & NS \\
\hline Cerebral & $1(2.5)$ & $3(0.4)$ & \\
\hline Digestive & $3(7.6)$ & $35(7.9)$ & \\
\hline Hemoptysis & 0 & $7(1.5)$ & \\
\hline Urinary & 0 & $11(2.4)$ & \\
\hline Hematoma & 0 & $13(2.8)$ & \\
\hline Vascular & $1(2.5)$ & $9(1.9)$ & \\
\hline ENT & $1(2.5)$ & $1(0.2)$ & \\
\hline TIMI class & $N=6$ & $N=79$ & NS \\
\hline Major & $2(5.1)$ & $4(0.8)$ & \\
\hline Minor & $2(5.1)$ & $38(8.5)$ & \\
\hline Minimal & $2(5.1)$ & $37(8.2)$ & \\
\hline Bleeding on TT regimen & & 40 & \\
\hline VKA & 2 & 20 & \\
\hline Rivaroxaban & 2 & 8 & NS \\
\hline Apixaban & 1 & 11 & \\
\hline Dabigatran & 1 & & \\
\hline
\end{tabular}

VASP: Vasodilator-stimulated phosphoprotein; AF: Atrial fibrillation; INR: International normalized ratio MACCE: Major adverse cardiac and cerebrovascular events; MI: Myocardial infarction; PCI: Percutaneous coronary intervention; PPI: proton pump inhibitor; VASP: Vasodilator-stimulated phosphoprotein; TIMI: Thrombolysis in myocardial infarction; VKA: Vitamin K antagonist; VTED: Venous thromboembolism disease; TT: Triple therapy.

\section{Discussion}

Our results provide the largest dataset of PRI in ACS patients undergoing PCI and receiving TAT. Our study failed to demonstrate that VASP assays could predict the clinical outcome of patients in this population. The differences between the two groups (HTPR, LTPR or not) regarding events were not statistically significant but this observation needs to be confirmed using a larger study population. The results were surprising, as it is known that HTPR is associated with ischemic events and LTPR is associated with bleeding [12,13].

\subsection{PRI and MACCE or Bleedings at 6 Months}

Several reasons may explain why PRI assessment did not predict the occurrence of MACCE or bleeding at six months in our study. First, data on cutoff values for VASP index in a setting of TAT regimen are limited, and it may well be that cutoff values that best determine patients risk for ischemia or bleeding may differ in this cohort compared with values obtained in patients with antiplatelet treatment alone [17]. Another possible reason for the negative results may be the assay used for the determination of PRI [18,19]. Danese et al. assessed the relationships between the pharmacokinetic and pharmacodynamic properties of clopidogrel, with the aim of identifying which is the best PFT [20]. The VASP 
assay was strongly influenced by the peak concentration of clopidogrel active metabolite. Timely performed VASP-P test may be the best marker for clopidogrel bioavailability and seems to be of potential usefulness in clinical studies aimed to identify the thrombotic and hemorrhagic risk associated with clopidogrel administration. Conversely, for Helten et al. multiplate impedance aggregometry could possibly be the best assay for antiplatelet medication tailoring at present [21]. In 2019, key opinions leaders summarized the updated expert consensus recommendations for the selective use of PFT in patients undergoing PCI [22]. Patients receiving OAC and antiplatelet treatment were described as an area of unmet need [19-23]. The only study analyzing PRI via VASP assays in patients on TAT regimen was conducted by Hu et al. [24]. In this study, 503 patients receiving TAT were randomized into two groups: the first group received dose titration of clopidogrel based on PRI, and the second group received a standard dose of $75 \mathrm{mg}$ of clopidogrel. The results showed that the group receiving adjusted therapy based on PRI had significantly less MACCE at one year ( $2.5 \%$ vs. $5.0 \%, p=0.02)$ without an increase in major bleeding $(3.0 \%$ vs. $2.8 \%$ ). However, these patients had a significantly higher rate of minor bleeding at 1 year than patients in the standard group $(15.3 \%$ vs. $9.7 \%, p=0.03)$.

\subsection{VASP and TAT Regimen}

To the best of our knowledge, this is the first prospective study assessing VASP assay in patients with TAT regimen. Compared with our study, Hu et al.'s population had a higher rate of MACCE (14.5\% of patients) because their endpoints concerned target vessel revascularization (TVR) [22]. TVR is not influenced by antithrombotic regimen and it was not used in RCT- AF PCI patients [3]. Patients were younger (respectively, 63 years versus 80 years) with a lower CHA2DS2VASc score (3.7 versus 4.72 in our study). This study was underpowered with a relatively small sample of patients recruited in this low risk population (stable CAD scheduled for PCI with warfarin stopped three days before PCI). Third, only AF patients were considered as eligible. It is uncertain if PRI values should be used in all TAT -treated subjects. Fourth, the only anticoagulant treatment prescribed was VKA. The authors did not evaluate the effects of the new DOACs. Fifth, the duration of TAT therapy was a fixed duration of three months (instead of six months in our study) followed by a fixed duration of DAPT with VKA.

Conversely, our study was conducted in real life, with VKAs agents and DOACs prescribed in a high-risk population (ACS patients, half of them were on HTPR for the ischemic risk, $\mathrm{CHA}_{2} \mathrm{DS}_{2} \mathrm{VASc}$ score 4.72 for the embolism risk and HAS-BLED score between 2 and 3 for bleeding risk). In addition, more than $50 \%$ of the patients in our study were treated with DOACs, and the median duration of TAT was 3.51 months which is quite different from Hu's study [24]. Our results are consistent with recent RCTs with a 17\% reported rate of bleeding events [3]. In our work, half of patients had HTPR, and numerous studies have shown that up to $40 \%$ of patients receiving clopidogrel developed HTPR [3].

In RCT, bleeding events were related to the duration and intensity of antiplatelet therapy [3] in DAPT. In our series, most bleeding events occurred within the first month because of INR imbalance issues. Ischemic events risk is increased within the first months after ACS as described in the AF-PCI trial. The high rate of MACCE early after PCI as observed in our work was confirmed in recent studies [3]. Our study confirms that DOACs showed the most favorable safety profile in TAT patients in comparison with VKA use. In our study more than half of MACCE and major bleeding occurred in the VKA group whether patient had HTPR or LTPR. In agreement with RCT trials, our study showed that DOAC seems to have a favorable efficacy risk profile with significant reduction in intracranial hemorrhages [25].

The recent nationwide Danish cohort study was unable to ascertain the clinical benefit of DOACs use because TAT regimen with VKA or DOAC was only prescribed in 1\% and $0.3 \%$ of patients, respectively. However, further studies are needed to ascertain the superiority of DOAC over VKA in TAT regimen. Our observational data suggest that the incidence of serious anticoagulant related gastrointestinal bleeding is higher for rivaroxaban than for 
dabigatran and apixaban. This is consistent with previous studies [26,27]. One explanation may be that rivaroxaban is given once daily and the relative peak plasma concentrations are greater than those for other OAC [28]. When choosing OAC in TAT regimen, practitioners should assess the risk of gastrointestinal bleeding. In contemporary practice, our study as with the others RCT trials confirmed that MACCE are five times less common than bleeding occurrences [3]. The higher incidence of MACCE in our VKA group may be explained by the interaction between VKA and clopidogrel. It has been shown than clopidogrel and VKA agents cause an intra-drug interaction resulting in an attenuated antiplatelet effect of clopidogrel. VKA significantly alters the in vivo biotransformation of clopidogrel into its active thiol metabolite [19]. Our results are consistent with this observation. Among the 201 patients on VKA, the mean VASP index was $55.31 \%$ in VKA agents versus $44.62 \%$ in the DOAC group (data not shown). According with the guidelines, lower-dose rivaroxaban regimen (15 $\mathrm{mg}$ a day) was used and this may explain the increase of MACCE comparing with full-dose regimen of apixaban and dabigatran [3].

\subsection{Strength and Limitation}

The study is underpowered, as only 38 ischemic events were identified and the majority of these were all-cause deaths ( $n=18$, i.e., $47 \%$ of overall ischemic events).

This subject seems out of date because TAT has been shortened to one month and DOACs are strongly recommended [3]. Nevertheless, still many patients represent a challenge for interventional cardiologist because they present a high ischemic risk post PTCA (left main disease, history of stent thrombosis, etc.) and require several months of TAT [3]. Some practitioners continue to use PFT to guide de-escalation. This article should warn them about this falsely reassuring practice.

In our series, patients have a particularly severe profile when compared to the literature: older (mean age 78 years old), elevated $\mathrm{CHA}_{2} \mathrm{DS}_{2} \mathrm{VASc}$ score and high proportion of comorbidities. In addition, almost $60 \%$ of patients were treated with DOACs, and the median duration of TAT was 3.5 months, resulting in important differences with the data in the literature [1-6,24].

Many potential factors including clinical variables such as diabetes mellitus, renal failure, drug interactions and non-adherence to treatment may influence ischemic risk in ACS patients on TAT with clopidogrel due to its heterogeneous and unpredictable antiplatelet effect [3]. Genetic polymorphism of genes coding for clopidogrel absorption in intestines exert a crucial role in clopidogrel bioactivation. Clopidogrel is a prodrug, and ATP-binding cassette sub-family B member 1 (ABCB1) is involved in the intestinal absorption of the drug $[29,30]$.

The optimal timing of testing with relationship to the PCI procedure remains a topic of debate. As for other biomarkers in cardio-vascular medicine such as troponins and pro-brain natriuretic peptide, a single test is a representative snapshot of the status quo for the time point when it is determined [22]. In addition, platelet reactivity is enhanced in the earlier phases of ACS and diminishes over time [22].

One strategy to resolve the question as to whether this genetic variation is involved in outcomes might be the method of Mendelian randomization.

Despite all these limitations, this is the first and the largest cohort of ACS patients on TAT regimen with PRI assessment in the real world. The sample of patients was close to the sub-groups in RCT, but in a high-risk population with every OAC (including DOAC) in all TAT-treated subjects (including mechanical heart valves and venous thromboembolism).

\section{Conclusions}

The optimal antithrombotic therapy after PCI in patients receiving long-term OAC remains controversial. RCTs have demonstrated that dual therapy (DOAC plus clopidogrel) significantly reduces bleeding risk in comparison to TAT regimen with non-significant trends towards higher risk of MI. Data were thus needed to help decision making to 
implement de-escalation strategy from TAT to dual therapy or to shorten the TAT regimen duration.

The use of VASP assays has been suggested to be a useful tool to help clinical decision, but our study, from the largest sample size to date, does not support this practice. However, our findings strongly suggest that DOAC should be prioritized.

Author Contributions: Conceptualization: J.G., Y.C. and F.P.; Methodology: J.G., H.Y., N.R., M.M. and M.Z. Software: N.R., M.M. and M.Z. Validation: J.G. and F.P.; Formal analysis: J.G., F.P., E.D.M. and L.C.-J. Investigation: L.B., M.L. and F.P.; Resources: L.B., M.L. and F.P. Data curation: N.R., M.M. and M.Z.; Writing—original draft preparation: J.G. and F.P.; Writing—review and editing: J.G. and F.P. Supervision: F.P.; Project administration: F.P. All authors have read and agreed to the published version of the manuscript.

Funding: This research received no external funding.

Institutional Review Board Statement: The protocol was approved by local ethic committees and in accordance with the Declaration of Helsinki [Prospective Real-world Registry Describing Treatment Regimens (PRAETORIAN) NCT03942913].

Informed Consent Statement: Informed consent was obtained from all subjects involved in the study.

Data Availability Statement: The data presented in this study are available on request from the corresponding author.

Conflicts of Interest: The authors declare no conflict of interest.

\section{References}

1. Morita, Y.; Hamaguchi, T.; Yamaji, Y.; Hayashi, H.; Nakane, E.; Haruna, Y.; Haruna, T.; Hanyu, M.; Inoko, M. Temporal trends in prevalence and outcomes of atrial fibrillation in patients undergoing percutaneous coronary intervention. Clin. Cardiol. 2019, 43, 33-42. [CrossRef]

2. Capodanno, D.; Alfonso, F.; Levine, G.N.; Valgimigli, M.; Angiolillo, D.J. Dual antiplatelet therapy: Appraisal of the ACC/AHA and ESC focused updates. J. Am. Coll. Cardiol. 2018, 72, 103-119.

3. Capodanno, D.; Huber, K.; Mehran, R.; Lip, G.Y.H.; Faxon, D.P.; Granger, C.B.; Vranckx, P.; Lopes, R.D.; Montalescot, G.; Cannon, C.P.; et al. Management of Antithrombotic Therapy in Atrial Fibrillation Patients Undergoing PCI: State-of-the-Art. J. Am. Coll. Cardiol. 2019, 74, 83-99. [CrossRef] [PubMed]

4. Singh, M.; Sporn, Z.A.; Schaff, H.V.; Pellikka, P.A. ACC/AHA Versus ESC Guidelines on Prosthetic Heart Valve Management: JACC Guideline Comparison. J. Am. Coll. Cardiol. 2019, 73, 1707-1718. [CrossRef] [PubMed]

5. Douketis, J.D. The 2016 American College of Chest Physicians treatment guidelines for venous thromboembolism: A review and critical appraisal. Intern. Emerg. Med. 2016, 11, 1031-1035. [CrossRef] [PubMed]

6. Lamberts, M.; Gislason, G.H.; Olesen, J.B.; Kristensen, S.L.; Olsen, A.-M.S.; Mikkelsen, A.; Christensen, C.B.; Lip, G.Y.; Køber, L.; Torp-Pedersen, C.; et al. Oral Anticoagulation and Antiplatelets in Atrial Fibrillation Patients After Myocardial Infarction and Coronary Intervention. J. Am. Coll. Cardiol. 2013, 62, 981-989. [CrossRef]

7. Neumann, F.-J.; Sousa-Uva, M.; Ahlsson, A.; Alfonso, F.; Banning, A.P.; Benedetto, U.; Byrne, R.A.; Collet, J.-P.; Falk, V.; Head, S.J.; et al. 2018 ESC/EACTS Guidelines on myocardial revascularization. Eur. Heart J. 2018, 40, 87-165. [CrossRef]

8. Tantry, U.S.; Bonello, L.; Aradi, D.; Price, M.J.; Jeong, Y.-H.; Angiolillo, D.J.; Stone, G.W.; Curzen, N.; Geisler, T.; Berg, J.T.; et al. Working Group on On-Treatment Platelet Reactivity. Consensus and Update on the Definition of On-Treatment Platelet Reactivity to Adenosine Diphosphate Associated With Ischemia and Bleeding. J. Am. Coll. Cardiol. 2013, 62, 2261-2273. [CrossRef]

9. Aradi, D.; Komócsi, A.; Vorobcsuk, A.; Rideg, O.; Tőkés-Füzesi, M.; Magyarlaki, T.; Horváth, I.G.; Serebruany, V.L. Prognostic significance of high on-clopidogrel platelet reactivity after percutaneous coronary intervention: Systematic review and metaanalysis. Am. Heart J. 2010, 160, 543-551. [CrossRef]

10. Vranckx, P.; Verheugt, F.W.; De Maat, M.P.; Ulmans, V.A.W.; Regar, E.; Smits, P.; Berg, J.M.T.; Lindeboom, W.; Jones, R.L.; Friedman, J.; et al. A randomised study of dabigatran in elective percutaneous coronary intervention in stable coronary artery disease patients. EuroIntervention 2013, 8, 1052-1060. [CrossRef]

11. Bonello, L.; Mancini, J.; Pansieri, M.; Maillard, L.; Rossi, P.; Collet, F.; Jouve, B.; Wittenberg, O.; Laine, M.; Michelet, P.; et al. Relationship between post-treatment platelet reactivity and ischemic and bleeding events at 1-year follow-up in patients receiving prasugrel. J. Thromb. Haemost. 2012, 10, 1999-2005. [CrossRef]

12. Sibbing, D.; Steinhubl, S.R.; Schulz, S.; Schömig, A.; Kastrati, A. Platelet aggregation and its association with stent thrombosis and bleeding in clopidogrel-treated patients: Initial evidence of a therapeutic window. J. Am. Coll. Cardiol. 2010, 56, 317-318. [CrossRef] [PubMed] 
13. Aradi, D.; Kirtane, A.; Bonello, L.; Gurbel, P.A.; Tantry, U.S.; Huber, K.; Freynhofer, M.K.; Berg, J.T.; Janssen, P.; Angiolillo, D.J.; et al. Bleeding and stent thrombosis on P2Y12-inhibitors: Collaborative analysis on the role of platelet reactivity for risk stratification after percutaneous coronary intervention. Eur. Heart J. 2015, 36, 1762-1771. [CrossRef]

14. Alpert, J.S.; Jaffe, A.S.; Chaitman, B.R.; Bax, J.J.; Morrow, D.A.; White, H.D. Executive Group on behalf of the Joint European Society of Cardiology (ESC)/American College of Cardiology (ACC)/American Heart Association (AHA)/World Heart Federation (WHF) Task Force for the Universal Definition of Myocardial Infarction Fourth Universal Definition of Myocardial Infarction. J. Am. Coll. Cardiol. 2018, 72, 2231-2264.

15. Whiteley, W.N.; Slot, K.B.; Fernandes, P.; Sandercock, P.; Wardlaw, J. Risk factors for intracranial hemorrhage in acute ischemic stroke patients treated with recombinant tissue plasminogen activator: A systematic review and metaanalysis of 55 studies. Stroke 2012, 43, 2904-2909. [CrossRef] [PubMed]

16. Kikkert, W.J.; van Geloven, N.; van der Laan, M.H.; Vis, M.M.; Baan, J., Jr.; Koch, K.T.; Peters, R.J.; de Winter, R.J.; Piek, J.J.; Tijssen, J.G.P.; et al. The prognostic value of bleeding academic research consortium (BARC)-defined bleeding complications in ST-segment elevation myocardial infarction: A comparison with the TIMI (thrombolysis in myocardial infarction), GUSTO (global utilization of streptokinase and tissue plasminogen activator for occluded coronary arteries), and ISTH (international society on thrombosis and Haemostasis) bleeding classifications. J. Am. Coll. Cardiol. 2014, 63, 1866-1875.

17. Chatterjee, S.; Sharma, A.; Uchino, K.; Biondi-Zoccai, G.; Lichstein, E.; Mukherjee, D. Rivaroxaban and risk of myocardial infarction: Insights from a meta-analysis and trial sequential analysis of randomized clinical trials. Coron. Artery Dis. 2013, 24, 628-635. [CrossRef]

18. Bahit, M.C.; Lopes, R.D.; Wojdyla, D.M.; Hohnloser, S.H.; Alexander, J.H.; Lewis, B.S.; Aylward, P.E.; Verheugt, F.W.; Keltai, M.; Diaz, R.; et al. Apixaban in patients with atrial fibrillation and prior coronary artery disease: Insights from the ARISTOTLE trial. Int. J. Cardiol. 2013, 170, 215-220. [CrossRef]

19. Sibbing, D.; Von Beckerath, N.; Morath, T.; Stegherr, J.; Mehilli, J.; Sarafoff, N.; Braun, S.; Schulz, S.; Schömig, A.; Kastrati, A. Oral anticoagulation with coumarin derivatives and antiplatelet effects of clopidogrel. Eur. Heart J. 2010, 31, 1205-1211. [CrossRef]

20. Danese, E.; Fava, C.; Beltrame, F.; Tavella, D.; Calabria, S.; Benati, M.; Gelati, M.; Gottardo, R.; Tagliaro, F.; Guidi, G.C.; et al. Relationship Between Pharmacokinetics and Pharmacodynamics of Clopidogrel in going Percutaneous Coronary Intervention: Comparison Between VASP Assay and Multiple Electrode Aggregometry. J. Thromb. Haemost. 2016, 14, 282-293. [CrossRef]

21. Helten, C.; Naguib, D.; Dannenberg, L.; Pöhl, M.; Ayhan, A.; Hohlfeld, T.; Levkau, B.; Kelm, M.; Zeus, T.; Polzin, A. Platelet function testing: Dead or alive. J. Thromb. Haemost. 2018, 16, 984-986. [CrossRef] [PubMed]

22. Sibbing, D.; Aradi, D.; Alexopoulos, D.; Berg, J.T.; Bhatt, D.L.; Bonello, L.; Collet, J.-P.; Cuisset, T.; Franchi, F.; Gross, L.; et al. Updated Expert Consensus Statement on Platelet Function and Genetic Testing for Guiding P2Y12 Receptor Inhibitor Treatment in Percutaneous Coronary Intervention. JACC Cardiovasc. Interv. 2019, 12, 1521-1537. [CrossRef] [PubMed]

23. Sibbing, D.; Angiolillo, D.J. Reply: Platelet Function and Genetic Testing in Patients Receiving Oral Anticoagulation and Antiplatelet Treatment: An Area of Unmet Need. J. Am. Coll. Cardiol. Intv. 2019, 12, 1868-1869. [CrossRef] [PubMed]

24. Hu, C.; Zhang, X.; Liu, Y.; Gao, Y.; Zhao, X.; Zhou, H.; Luo, Y.; Liu, Y.; Wang, X. Vasodilator-stimulated phosphoprotein-guided Clopidogrel maintenance therapy reduces cardiovascular events in atrial fibrillation patients requiring anticoagulation therapy and scheduled for percutaneous coronary intervention: A prospective cohort study. BMC Cardiovasc. Disord. 2018, 18, 120. [CrossRef] [PubMed]

25. Ruff, C.T.; Giugliano, R.P.; Braunwald, E.; Hoffman, E.B.; Deenadayalu, N.; Ezekowitz, M.D.; Camm, A.J.; Weitz, J.I.; Lewis, B.S.; Parkhomenko, A.; et al. Comparison of the efficacy and safety of new oral anticoagulants with warfarin in patients with atrial fibrillation: A meta-analysis of ramdomised trials. Lancet 2014, 383, 955-962. [CrossRef]

26. Van Rein, N.; Heide-Jørgensen, U.; Lijfering, W.M.; Dekkers, O.M.; Sørensen, H.T.; Cannegieter, S.C. Major bleeding rates in atrial fibrillation patients on single, dual, or triple antithrombotic therapy. Circulation 2019, 139, 775-786. [CrossRef] [PubMed]

27. Ray, W.A.; Chung, C.P.; Murray, K.T.; Smalley, W.E.; Daugherty, J.R.; Dupont, W.D.; Stein, C.M. Association of Oral Anticoagulants and Proton Pump Inhibitor cotherapy With Hospitalization for Upper Gastrointestinal Tract Bleeding. JAMA 2018, 320, $2221-2230$. [CrossRef]

28. Gong, I.Y.; Kim, R.B. Importance of Pharmacokinetic Profile and Variability as Determinants of Dose and Response to Dabigatran, Rivaroxaban, and Apixaban. Can. J. Cardiol. 2013, 29, S24-S33. [CrossRef]

29. Taubert, D.; Von Beckerath, N.; Grimberg, G.; Lazar, A.; Jung, N.; Goeser, T.; Kastrati, A.; Schömig, A.; Schömig, E. Impact of P-glycoprotein on clopidogrel absorption. Clin. Pharmacol. Ther. 2006, 80, 486-501. [CrossRef] [PubMed]

30. Simon, T.; Verstuyft, C.; Mary-Krause, M.; Quteineh, L.; Drouet, E.; Méneveau, N.; Steg, P.G.; Ferrières, J.; Danchin, N.; Becquemont, L. Genetic Determinants of Response to Clopidogrel and Cardiovascular Events. N. Engl. J. Med. 2009, 360, 363-375. [CrossRef] 